\title{
Morphosyntactic abilities and reading skills in Arabic speaking Egyptian children with poor academic achievement
}

\author{
Original \\ Article \\ Dalia Mostafa Osman ${ }^{1}$, Rasha Farouk Safwat ${ }^{2}$, Ainulhayah Hamed Mohammady \\ Asmaa Ahmed Abdel Hamid ${ }^{4}$
}

Phoniatric Unit, ENT Department, ${ }^{1,2,4}$ Cairo University, ${ }^{3}$ Menufeya University, Egypt.

\begin{abstract}
Background: The most common learning disability affecting academic performance is Dyslexia. Linguistic abilities like morphological awareness, semantic and syntactic abilities play a key role in reading development too. The paper aimed to study the extent and properties of morphological aspects and syntactic functioning in relation to reading skills in a group of Egyptian children having poor academic achievement as for designing suitable intervention plans for these children.

Patients and Methods: Our study aimed to assess the extent and properties of morphological aspects and syntactic functioning in relation to reading skills in 40 Egyptian Arabic speaking children of the same socioeconomic state and age range from 6 years to 8 years with poor academic performance. The children divided into two groups were subjected to a protocol of assessment applied in Phoniatrics Unit Kasr Al aini Hospital, Arabic Dyslexia Assessment Test and language skills assessment by Receptive Expressive Arabic Language scale- REAL scale were applied.

Results: All children with poor scholastic achievement were found to be at risk for developing dyslexia if not suffering from it. They suffered from below average level of performance in receptive, expressive and total language. All language parameters showed significant correlation with rapid naming. Below average morpho-syntactic abilities of children with poor scholastic achievement significantly correlated with tests of attainment (except for spelling) and diagnostic tests (except for bead threading).

Conclusion: Our study supports that language disability underlies poor scholastic achievement.
\end{abstract}

Key Words: Arabic Egyptian children, dyslexia, language, morpho-syntactic abilities, poor scholastic achievement.

Received: 11 February 2021, Accepted: 19 July 2021

Corresponding Author: Ainulhayah Hamed Mohammady, MD, Phoniatric Unit, ENT Department, Menufeya University Hospitals, Egypt., Tel.: 048 3651001, E-mail: e3yoong@gmail.com

ISSN: 2090-0740, 2021

\section{INTRODUCTION}

Learning disability is a heterogeneous group of disorders that is manifested by difficulties in the acquisition and use of listening, speaking, reading, writing, reasoning or mathematical abilities. ${ }^{[1]}$ Dyslexia is "the restricted ability or inability to process series of printed words, phrases or sentences"[2].

Although the precise nature and origin of dyslexia are still in debate, results from studies ascribing literacy problems in dyslexics to a phonological dysfunction ${ }^{[3,4]}$.

Morphological awareness skills, have been reported to be strongly associated with reading achievement (i.e., reading at word-level and reading comprehension) eitherindyslexics or non-dyslexics ${ }^{[5,6]}$. There is also evidence that dyslexic individuals have poorer morphological processing skills in comparison to their chronological age peers ${ }^{[7]}$. However, Oral language abilities in dyslexia have received little consideration in most studies. Some researchers like Storch \&Whitehurst $(2002)^{[8]}$ noted that reading development and acquisition is complicated and that components of oral language, such as semantic and syntactic abilities, play a key role in reading development. To better understand the acquisition of efficient reading it is important to explain how the various components related to reading development - oral language, word reading, and reading comprehension - come together and interact.

Dyslexia in Arabic language is distinct from English dyslexia. There were few researches to address dyslexia in Arabic speaking. In Arabic, there are numerous factors which could lead to the development of dyslexia among Arabic speakers. This knowledge is not only crucial to a linguistic typology of dyslexia, but can also be used to test theoretical causes (morpho-syntactic issues in developmental dyslexia) may also be investigated.

Work is required to explain the theoretical explanation of morpho-syntactic Problems in developmental dyslexia.

Work is required to explain the relationship between language competences and the development of reading. 


\section{AIM OF THE STUDY :}

The aim of this study is to study the extent and properties of morphological aspects and syntactic functioning in relation to reading skills in a group of Egyptian Arabic speaking children having poor academic achievement. This would be of great help in designing suitable intervention plans for these children.

\section{PATIENTS AND METHODS:}

\section{Subjects:}

This study was conducted on a sample of 40 Egyptian Arabic speaking children of the same socioeconomic state divided into two groups. The first group (24 males and 4 females) aged from 6 years 6 months to 7 years 8 months (mean 7 years) while the second group ( 7 males and 5 females) aged from 6 years to 6 years 5 months (mean 6 years), who attended the Phoniatrics clinic in Menoufeya University Hospital in Mansheyat Sultan during the period from September 2018 to March 2019.

These children had normal cognitive abilities, no physical handicaps and most of them had received general education prior to testing. The referral complaint for 32 child was poor academic performance, while the referral complaint for 8 children was phonological disorders and on taking history of academic achievement the parent complained of poor academic performance.

Patients who were excluded from the study had any of the following:

1- Hearing impairment.

2- Structural or motor speech problems.

3- Symptoms of psychological disorders or behavior disabilities.

4- Neurological disorders.

5- Autism spectrum disorders

6- Sever Inconsistency in school attendance

\section{Method:}

\section{Assessment protocol:}

Every case was subjected to the following protocol of assessment D- Elementary diagnostic procedures:

I-Complete history taking.

- 1-Personal history.
- 2-Family history

- 3-Prenatal and natal history

- 4-Developmental history.

- 5-History of early childhood illness.

II-General examination

III-Vocal tract examination

IV-Ear and nose examination

V-Neurological examination

\section{B- Psychometric evaluation using}

Stanford Binet Intelligence Scale " $4^{\text {th }}$ Arabic version" for determination of mental age. Only children with an IQ 80 or above participated in the study.

\section{C- Communicative assessment}

Assessing Arabic language skills in each child under study using the Receptive

Expressive Arabic Language scale- REAL scale ${ }^{[10]}$ include the following subtests: o Receptive Vocabulary o Sentence Comprehension o Verbal Categorization I o Verbal categorization 2 o Comprehending Orally Presented Paragraphs o Expressive Vocabulary o Morpho-syntax o Sentence Repetition o Forming Sentences I

o Forming Sentences 2 (N.B for Verbal Categorization Expressive I and Verbal categorization Expressive 2, the examiner uses the same pictures and verbal stimuli included in the receptive part of these subtests).

\section{REAL Scale items have been classified into:}

I-Receptive subtests include:

- $\quad$ Receptive Vocabulary (RV)

- $\quad$ Sentence Comprehension (SC

- Understanding Oral Instructions (UOI):

- Comprehending Orally Presented Paragraphs (COPP)

- Verbal Categorization Receptive1 (VCRI)

- Verbal Categorization Receptive 2(VCR2) 


\section{II-Expressive subtests include:}

- $\quad$ Expressive Vocabulary (EV)

- $\quad$ Forming Sentences 1 (FS 1)

- $\quad$ Forming Sentences 2 (FS2)

- $\quad$ Sentence Repetition (SR)

- Morpho-syntax (MS)

- Verbal Categorization Expressive 1 (VCEI)

- Verbal Categorization Expressive 2 (VCE2)

\section{D- Learning disabilities assessment:}

Arabic Dyslexia Assessment Test ${ }^{[11]}$ was applied for each child in the study. The test is composed of eleven items. These are:

1. *Rapid naming test.

2. *Bead threading.

3. *One minute reading.

4. *Postural stability.

5. *Phonemic segmentation.

6. *Two minute spelling.

7. *Backward digit span.

8. *Nonsense passage reading.

9. *One minute writing.

10. *Verbal fluency.

11. *Semantic fluency.

After finding the score key appropriate for the child's age in the age group $\left(6 \frac{1}{2}-7\right.$ years 8 months $)$, each item was given one of the following scores: $(+),(0),(-),(--)$, (---). Finally, the test yielded an at risk quotient. If a child's at risk quotient was $\geq 1$, this was a strong evidence of being at risk of dyslexia.

Although the test score key appropriate for the child's age starts from age $6 \frac{1}{2}$ years, yet the test could be conducted to children less than 6 years 6 months up to 6 years as mentioned in the manual of the Arabic Dyslexia Assessment Test.
RESULTS:

Table (1) The group consisted of 28 child (24 males and 4 females) aged from 6 years 5 months to 7 years 8 months (mean 7 years). Their IQ ranged between 80 (lowest) and 106 (highest) according to the Stanford Binnet fourth edition test (mean 87).

Table (4) Morpho-Syntax showed significant negative correlation with each of rapid naming and postural stability, while it showed significant positive correlation with one min reading, Phonemic segmentation, Non sense passage reading, 1 min writing and verbal fluency

Table (5) shows that:

- RLS (receptive language skills) showed significant negative correlation with each of rapid naming and postural stability, while it showed significant positive correlation with one min reading, Phonemic segmentation, 2 min spelling, backward digit span, 1 min writing and verbal fluency.

- ELS (expressive language skills) showed significant negative correlation with each of rapid naming and postural stability, while it showed significant positive correlation with one min reading, Phonemic segmentation, 2 min spelling, backward digit span, non -sense passage reading, 1 min writing, verbal fluency and semantic fluency

- $\quad$ TLS showed significant negative correlation with each of rapid naming and postural stability, while it showed significant positive correlation with one min reading, Phonemic segmentation, 2 min spelling, backward digit span, non- sense passage reading, $1 \mathrm{~min}$ writing,verbal fluency and semantic fluency.

Table (6) showed no significant difference between the two groups (1 and 2) in any of the REAL scale subtests. It also shows that both groups suffer from below average scores in Sentence Comprehension (SC), Understanding Oral Instructions (UOI) and Comprehending Orally Presented Paragraphs (COPP) in the receptive subtests and in Expressive Vocabulary (EV), Sentence Repetition (SR), Verbal categorization expressive1 and Forming Sentences 2 (FS2) in the expressive subtests. While group 1 only suffered from below average score in Morpho-syntax (MS)

Table (7) showed no significant difference between both groups (1 and 2) in the scaled language scores of the REAL scale. Yet both groups show below average level of Receptive, Expressive and Total language. 
Table 1: Patients characteristics of Group 1:

\begin{tabular}{ll}
\hline Variable & Mean \pm SD, range \\
\hline Age in months & $84.57 \pm 5.71,77.0-94.0$ \\
& No. $(\%)$ \\
Gender: & \\
Male: & $24(85.7)$ \\
Female: & $4(14.3)$ \\
IQ & $87.21 \pm 6.88,80.0-106.0$ \\
\hline
\end{tabular}

Table 2: Comparison between Poor scholastic achievement and Phonological disorders sub groups according to referral complaint in the results of the REAL scale subtests applied to group1

\begin{tabular}{|c|c|c|c|c|}
\hline & $\begin{array}{c}\text { Poor scholastic } \\
\text { achievement }(n=23) \\
\text { Mean } \pm \text { SD }\end{array}$ & $\begin{array}{l}\text { Phonological } \\
\text { disorders }(n=5) \\
\text { Mean } \pm \text { SD }\end{array}$ & Mann Whitney test & Pvalue \\
\hline Receptive Vocabulary (RV) & $19.60 \pm 3.79$ & $20.00 \pm 7.17$ & 0.96 & 0.33 \\
\hline Sentence Comprehension (SC) & $41.87 \pm 7.04$ & $45.00 \pm 3.46$ & 0.89 & 0.37 \\
\hline Understanding Oral Instructions (UOI) & $32.82 \pm 10.56$ & $42.00 \pm 11.97$ & 1.56 & 0.37 \\
\hline Verbal Categorization Receptive1 & $14.56 \pm 4.93$ & $14.040 \pm 4.44$ & 0.18 & 0.85 \\
\hline Verbal Categorization & $7.69 \pm 4.23$ & $9.40 \pm 3.28$ & 0.93 & 0.35 \\
\hline \multicolumn{5}{|l|}{ Receptive2 } \\
\hline $\begin{array}{l}\text { Comprehending Orally Presented Paragraphs } \\
\text { (COPP) }\end{array}$ & $5.43 \pm 3.17$ & $7.80 \pm 3.49$ & 1.45 & 0.14 \\
\hline Expressive Vocabulary (EV) & $37.30 \pm 18.63$ & $44.80 \pm 19.39$ & 0.78 & 0.43 \\
\hline Morpho-syntax (MS) & $42.47 \pm 11.69$ & $47.40 \pm 11.23$ & 0.90 & 0.36 \\
\hline Sentence Repetition (SR) & $36.60 \pm 20.46$ & $54.60 \pm 20.67$ & 1.59 & 0.11 \\
\hline Verbal categorization expressive 1 & $11.13 \pm 4.34$ & $14.40 \pm 3.84$ & 1.59 & 0.11 \\
\hline Verbal categorization expressive 2 & $8.65 \pm 16.35$ & $7.60 \pm 2.96$ & 1.23 & 0.21 \\
\hline Forming Sentences 2 (FS2) & $10.69 \pm 9.36$ & $18.20 \pm 8.07$ & 2.01 & 0.04 \\
\hline Receptive language score & $113.69 \pm 33.96$ & $138.80 \pm 25.40$ & 1.86 & 0.06 \\
\hline Expressive Language score & $134.52 \pm 66.79$ & $307.60 \pm 77.14$ & 1.17 & 0.24 \\
\hline Total Language score & $252.56 \pm 66.79$ & $307.60 \pm 77.14$ & 1.35 & 0.17 \\
\hline
\end{tabular}

Table 3: Comparison between Poor scholastic achievement and Phonological disorders sub groups according to referral complaint in the results of the Arabic Dyslexia Quotient subtests applied to group 1 and their ADQ

\begin{tabular}{|c|c|c|c|c|}
\hline & $\begin{array}{c}\text { Poor scholastic } \\
\text { achievement }(n=23) \\
\text { Mean } \pm \text { SD }\end{array}$ & $\begin{array}{c}\text { Phonological } \\
\text { disorders }(n=5) \\
\text { Mean } \pm \text { SD }\end{array}$ & Mann Whitney test & Pvalue \\
\hline ADQ & $1.96 \pm 0.67$ & $1.70 \pm 0.64$ & 0.93 & 0.35 \\
\hline Rapid naming & $113.82 \pm 33.53$ & $105.20 \pm 56.28$ & 0.57 & 0.56 \\
\hline Bead threading & $3.26 \pm 1.42$ & $4.20 \pm 2.49$ & 0.89 & 0.37 \\
\hline One min reading & $4.30 \pm 5.56$ & $3.20 \pm 3.42$ & 0.09 & 0.92 \\
\hline Postural stability & $5.73 \pm 4.65$ & $3.80 \pm 2.16$ & 0.94 & 0.34 \\
\hline Phonemic segmentation & $3.47 \pm 2.87$ & $3.80 \pm 2.16$ & 0.39 & 0.69 \\
\hline 2 min spelling & $1.61 \pm 2.21$ & $1.40 \pm 1.34$ & 0.22 & 0.82 \\
\hline Backward digit span & $1.69 \pm 1.32$ & $2.40 \pm 1.67$ & 0.92 & 0.35 \\
\hline Non sense passage reading & $6.84 \pm 7.17$ & $11.30 \pm 11.82$ & 0.80 & 0.41 \\
\hline 1 min writing & $6.41 \pm 4.71$ & $7.20 \pm 4.32$ & 0.54 & 0.58 \\
\hline Verbal fluency & $2.34 \pm 3.00$ & $2.00 \pm 2.54$ & 0.00 & 1.00 \\
\hline Semantic fluency & $6.21 \pm 3.59$ & $7.80 \pm 3.49$ & 1.21 & 0.22 \\
\hline
\end{tabular}


Table 4: Correlation between Morphosyntax subtest results and the results of the Arabic Dyslexia Quotient subtests applied to group 1

\begin{tabular}{lll}
\hline & MS & \\
& $\mathrm{R}$ & P value \\
\hline Rapid naming & -0.58 & 0.001 \\
Bead threading & 0.34 & 0.06 \\
One min reading & 0.54 & 0.006 \\
Postural stability & -0.42 & 0.02 \\
Phonemic segmentation & 0.52 & 0.005 \\
2 min spelling & 0.35 & 0.06 \\
Backward digit span & 0.32 & 0.09 \\
Non sense passage reading & 0.44 & 0.01 \\
1 min writing & 0.38 & 0.04 \\
Verbal fluency & 0.71 & $<0.001$ \\
Semantic fluency & 0.33 & 0.08 \\
\hline
\end{tabular}

Table 5: correlation between Receptive language score (RLS), Expressive Language score (ELS) and Total language score (TLS) of the REAL scale and the results of the Arabic Dyslexia Quotient subtests applied to group 1

\begin{tabular}{lllllll}
\hline & RLS & & ELS & & TLS \\
& $\mathrm{r}$ & Pvalue & $\mathrm{r}$ & Pvalue & $\mathrm{r}$ & Pvalue \\
\hline Rapid naming & -0.46 & 0.01 & -0.49 & 0.008 & -0.49 & 0.007 \\
Bead threading & 0.26 & 0.17 & 0.31 & 0.10 & 0.27 & 0.15 \\
One min reading & 0.53 & 0.004 & 0.51 & 0.005 & 0.56 & 0.002 \\
Postural stability & -0.51 & 0.005 & -0.56 & 0.002 & -0.57 & 0.001 \\
Phonemic segmentation & 0.44 & 0.01 & 0.55 & 0.002 & 0.54 & 0.003 \\
2 min spelling & 0.37 & 0.04 & 0.44 & 0.01 & 0.44 & 0.01 \\
Backward digit span & 0.40 & 0.03 & 0.37 & 0.04 & 0.37 & 0.04 \\
Non sense passage & 0.32 & 0.09 & 0.47 & 0.01 & 0.53 & 0.004 \\
reading & 0.68 & $<0.001$ & 0.43 & 0.02 & 0.39 & 0.03 \\
1 min writing & 0.45 & 0.01 & 0.76 & $<0.001$ & 0.79 & $<0.001$ \\
Verbal fluency & 0.35 & 0.06 & 0.47 & 0.01 & 0.49 & 0.04 \\
Semantic fluency & & & & & \\
\hline
\end{tabular}

Table 6: Comparison between the Descriptive statistics of the scaled scores of the REAL scale subtests applied to group 1 and group 2

\begin{tabular}{lcccc}
\hline & $\begin{array}{c}\text { Group 1 (n=28) } \\
\text { Mean } \pm \text { SD }\end{array}$ & $\begin{array}{c}\text { Group 2 (n=12) } \\
\text { Mean } \pm \text { SD }\end{array}$ & U Test & Pvalue \\
\hline Receptive Vocabulary (RV) & $7.28 \pm 2.25$ & $7.58 \pm 3.28$ & 0.03 & 0.97 \\
Sentence Comprehension (SC) & $6.25 \pm 2.97$ & $6.66 \pm 2.87$ & 0.46 & 0.64 \\
Understanding Oral Instructions (UOI) & $6.03 \pm 2.75$ & $6.25 \pm 2.22$ & 0.44 & 0.65 \\
Verbal Categorization Receptive1 & $7.64 \pm 3.27$ & $7.58 \pm 3.44$ & 0.01 & 0.98 \\
Verbal Categorization Receptive2 & $8.17 \pm 2.49$ & $8.91 \pm 1.16$ & 1.05 & 0.29 \\
Comprehending Orally Presented Paragraphs (COPP) & $5.83 \pm 3.10$ & $5.08 \pm 2.87$ & 0.59 & 0.55 \\
Expressive Vocabulary (EV) & $6.50 \pm 2.78$ & $6.58 \pm 1.83$ & 0.35 & 0.72 \\
Morpho-syntax (MS) & $6.92 \pm 3.09$ & $7.08 \pm 2.64$ & 0.10 & 0.91 \\
Sentence Repetition (SR) & $5.72 \pm 2.52$ & $6.58 \pm 1.92$ & 0.86 & 0.38 \\
Verbal categorization expressive 1 & $6.92 \pm 2.98$ & $6.83 \pm 2.72$ & 0.25 & 0.80 \\
Verbal categorization expressive 2 & $7.50 \pm 2.31$ & $8.50 \pm 1.00$ & 1.17 & 0.24 \\
Forming Sentences 2 (FS2) & $6.78 \pm 2.72$ & $6.75 \pm 2.23$ & 0.09 & 0.92 \\
\hline
\end{tabular}


Table 7: Comparison between the Descriptive statistics of the scaled language scores of the REAL scale applied to group 1 and group 2

\begin{tabular}{lllll}
\hline & $\begin{array}{l}\text { Group 1 }(\mathrm{n}=28) \\
\text { Mean } \pm \text { SD }\end{array}$ & $\begin{array}{l}\text { Group 2 }(\mathrm{n}=12) \\
\text { Mean } \pm \text { SD }\end{array}$ & U Test & Pvalue \\
\hline Receptive language score & $79.64 \pm 17.23$ & $83.08 \pm 11.84$ & 1.27 & 0.20 \\
Expressive Language score & $78.57 \pm 13.34$ & $80.41 \pm 11.21$ & 0.93 & 0.35 \\
Total Language score & $78.46 \pm 12.87$ & $81.91 \pm 11.37$ & 1.06 & 0.28 \\
\hline
\end{tabular}

\section{DISCUSSION}

The relation between language and Dyslexia has been studied in the current study. A negative correlation was found between Morpho-Syntax scores and rapid naming scores. Due to the method of scoring, the slower the rapid naming the higher the raw score. Thus our results indicate that the slower the speed of naming the weak the morpho-syntactic skills of the child and vice versa. This could be attributed to the "Integrative hypothesis", which is about the interactions between phonological processing, morphological/syntactic skill and naming speed as predictors of reading and spelling disability. According to which phonological, formal psycholinguistic skills (such as morphosyntactic skills) and cross-modal (visual-verbal) skills contribute to reading ${ }^{[12]}$.

A negative correlation was also found in the current study between MorphoSyntax subtest results and postural stability subtest-results of the Arabic Dyslexia assessment applied to group 1. The negative correlation in postural stability comes due to the method of scoring, as less the postural stability the higher the raw score. This indicates that the weaker the maintenance of postural stability the poorer the morpho-syntactic skills of the child. This could be due to the "cerebellar dysfunction" theory of dyslexia, as the cerebellum is important in the automatization of overlearned tasks and abnormal learning of the grapheme-phoneme correspondence could be a result of its dysfunction ${ }^{[13]}$.

A positive correlation was also found in our study between 1-minute reading and MorphoSyntax in group 1. This is attributed to the effect of morphological awareness on word pronunciation that is both phonological and semantic. As poor recognition of the morpho-syntactic markers that contributed to word meaning disrupts the semantic and phonological pathways to word identification skills needed for reading as postulated by Snowling et al. $(2006)^{[14]}$.

Our study showed positive correlation between phonemic segmentation and Morpho-Syntax raw scores in group 1 .This finding agree with a study that found that disruption of child acquisition of words and syntax is expected with early deficits in phonological processing skills ${ }^{[15]}$.
Another positive correlation between non-sense passage reading and Morpho- Syntactic skills was found in group 1 and group 2. In Arabic, dyslexic readers depend more on morphology whereas normal readers rely more on morphology only when the script is without vowels. Thus, the orthographic lexicon of dyslexic readers is richer than their phonological lexicon and they tend to depend on visualorthographic reading rather than reading mediated through phonology as reported by Abu-Rabia and Abu-Rahmoun (2012) ${ }^{[16]}$. As a result a poor lexicon of the dyslexic children may result from weak morphosyntactic skills thus increasing their difficulties with reading non-words compared to sight words.

In our current study there was a significant positive correlation between one - minute writing and MorphoSyntax scores in group 1. In Arabic language the knowledge of the morpho-orthographic structure of the word is an important facilitator of reading as reported by Abu-Rabia and Taha (2004) ${ }^{[17]}$. Accordingly, we assume that morpho-syntactic skills will affect the morpho-orthographic knowledge which in turn will affect literacy skills as writing.

In this study a positive correlation between MorphoSyntax and verbal fluency in group 1 was found. This is may be due to the effect of morphological awareness on the children ability to increase their vocabulary ${ }^{[18]}$.

We also found that total, receptive and expressive language scores showed negative correlation with rapid naming in both group 1 and group 2 . This could be explained by that a phonological deficit which is an underlying cause in both dyslexia and delayed language development ${ }^{[19]}$.

Receptive, expressive and total language scores showed negative correlation with postural stability in group 1. This could be explained that language impairment may result from an impaired system that will also affect learning of other procedural operations, such as motor skills and not only linguistic defect ${ }^{[20]}$.

The significant positive correlation in our study between one minute reading and total, receptive and expressive language of group 1 could be attributed to the relationship between reading and vocabulary. Both receptive and expressive vocabularies contribute 
significantly to pre-reading skills (phonology and print awareness). Receptive vocabulary is the primary foundation for building phonological awareness skills, whereas expressive vocabulary knowledge is a better predictor of word decoding skills as reported by Wise, et al., $(2007)^{[21]}$.

Significant positive correlation between phonemic segmentation and total, receptive and expressive language in group 1 in the current study is attributed to the phonological deficit hypothesis which proposes that phonological deficit underlie both delayed language development and dyslexia ${ }^{[22]}$.

A positive correlation was found between 2minute spelling and total, receptive and expressive language in group 1. This could be attributed to that language learning ability is affected significantly by metalinguistic skills such as orthographic knowledge as reported by Ginsberg et al., (2011) ${ }^{[23]}$.

The current study showed also positive correlation between the backward digit span (a test of working memory) and total, receptive and expressive language in group 1. This could be due to deficits in phonological short term memory would seem to impede verbal working memory required during spoken sentence comprehension thus affecting language according to a model of working memory supported by evidence from studies of children, adult participants, neuropsychological patients, and neuroimaging research like Gathercole et al., 2006 ${ }^{[24]}$.

There was a significant positive correlation between: one -minute writing and receptive language in both groups 1 and 2 . While in group 1 a positive correlation was found between, one -minute writing and total and expressive language too. This could be attributed to the different developmental stages students' progress through as they learn to read and write, specific to each skill as proposed by Fitzgerald \& Shanahan, $(2000)^{[25]}$.

Significantly positive correlation in the current study was found between verbal fluency and total, receptive and expressive language in group 1 . This is attributed to that the route to word identification is through knowing vocabulary.

The more details about words in the lexicon the more efficient encoding, organizing and retrieval of the phonological representations of words occurs ${ }^{[26]}$.

Significant positive correlation was found between semantic fluency and total and expressive language in both groups 1 and 2. While significant positive correlation with receptive language was found in group 2 only. This could be attributed to that the lexical semantics determines the acoustic and articulatory features relevant for a child's particular lexicon in a particular language. Nonsense passage reading and total and expressive language showed significant correlation in both groups 1 and 2 . This could be contributed to that reading non-sense words is the benchmark test of children's phonological decoding skill $^{[27]}$.

Relation between dyslexia and poor-scholastic achievement in the current study the Arabic Dyslexia assessment Test "At Risk Quotient" [ARQ] of group 1 had mean 1.91 with its standard deviation \pm 0.66 , lowest 1.0 highest 3.0 which shows that all group 1 children are at risk for developing dyslexia if not suffering from $\mathrm{it}^{[11]}$.

The current result could be attributed to that dyslexia is an invisible handicap, constitutes an important cause of poor school performance in children as postulated by Sukumaran, (2011). ${ }^{[28]}$

\section{Relation between language and poor-scholastic achievement:}

In the current study no significant difference was found between both groups ( 1 and 2 ) in the scaled language scores of the REAL scale and both groups showed below average level of Receptive, Expressive and Total language ${ }^{[10]}$. This could be explained by a large proportion of children who qualify as having delayed language development are either not identified or are identified in later school grades, based on problems with reading comprehension, as suggested from the evidence of several studies ${ }^{[29]}$.

In the current study both groups were found suffering from below average scores in the receptive subtests: Sentence Comprehension (SC), Understanding Oral Instructions (UOI) and Comprehending Orally Presented Paragraphs (COPP). This could be explained by that children with delayed language development, by definition, struggle with language comprehension; many also struggle with word reading, and most will struggle with reading comprehension.

Both groups of this study were found suffering from below average scores in the expressive subtests: Expressive Vocabulary (EV), Sentence Repetition (SR), Verbal categorization expressive1 (VCE1) and Forming Sentences 2 (FS2). While group 1 only suffered from below average score in Morpho-Syntax (MS).This could be attributed to that the children with dyslexia show poor word learning compared with typically developing peers, especially apparent when learning the phonological aspects of words ${ }^{[30]}$ 
The deficit in Sentence Repetition (SR) could point out an underling auditory processing disorder that can extend into reading and writing thus affecting scholastic achievement.

The comparison between the results of the REAL scale subtests of Poor scholastic achievement referral complaint children and Phonological disorders referral complaint children of group did not show any significant difference between the 2 subgroups except for "Forming Sentences 2" (FS2) of the expressive subtests was significantly higher in phonological disorders group than poor scholastic achievement group of group 1 and for Sentence Comprehension (SC), MorphoSyntax (MS) and Sentence Repetition (SR) were significantly higher in phonological disorders subgroup than poor scholastic achievement subgroup of group 2. this could be attributed to that the seek for help was elicited by phonological disorders, as the better language abilities of the phonological disorders subgroup masked the poor scholastic achievement in both groups 1 and 2 .

Thus we assume that the risk for later reading disability in phonological disorder is mediated by comorbid language disability. For the better language abilities of the phonological disorder subgroup masked their poor scholastic achievement rendering these children for risk of incomplete therapeutic intervention.

\section{STATE OF ETHICS}

The study protocol was approved by the ethical committee of Cairo University Hospital Research Committee. A written consent from children guardian was done before beginning of the study.

\section{CONFLICT OF INTEREST}

There are no conflicts of interest.

\section{REFERENCES}

1. National Joint Committee on Learning Disabilities. (1998). Operationalizing the NJCLD definition of learning disabilities for ongoing assessment in schools. Learning Disability Quarterly, 186-193.

2. Gósy, M. (1999). Psycholinguistics. Corvina. Budapest.

3. Siegel, L. S. (2008): Morphological awareness skills of English language learners and children with dyslexia. Topics in Language Disorders, 28(1), 15-27
4. Sprenger-Charolles, L., Colé, P. and W. Serniclaes (2006): Reading acquisition and developmental dyslexia. New York: Psychology Press.

5. Carlisle, J. F. (2000): Awareness of the structure and meaning of morphologically complex words: Impact on reading. Reading and Writing: An Interdisciplinary Journal, 12: 169-190

6. Nagy, W., Berninger, V., Abbott, R., Vaughan, K., \& Vermeulen, K. (2003). Relationship of morphology and other language skills to literacy skills in at-risk second-grade readers and at-risk fourth-grade writers. Journal of educational psychology, 95(4), 730-742.

7. Tsesmeli, S. N. and Seymour P. H. K. (2006): Derivational morphology and spelling in dyslexia. Reading and Writing, 19: 587-625.Ullman \& Pierpont (2005 8- Storch, S.A., and Whitehurst, G.J. (2002): Oral language and code-related precursors to reading: Evidence from a longitudinal structural model.

8. Developmental Psychology, 38(6), 934-947.

9. Melika, L. (1998): Stanford Binnet Intelligence Scale (4 ${ }^{\text {th }}$ Arabic version). Fourth Edition. Victor Kiorlos Publishing, Cairo.

10. Osman, D.A. (2014): REAL Scale (Receptive Expressive Arabic Language Scale), Print House, Cairo, Egypt

11. Aboras, Y., Abdou, R., Kozou, H. (2008) Development of an Arabic test for assessment of dyslexia in Egyptian children. Bulletin of Alexandria Faculty of Medicine; 44 (3): 653-662.

12. Plaza, M. (2001). The interaction between phonological processing, syntactic awareness and reading. First Language, 21, 3-24Rack (2017)

13. Nicolson, R. I. \& Fawcett, A. J., (2008). Dyslexia and the cerebellum. The SAGE handbook of dyslexia, 11-29.

14. Snowling, M., Bishop, D. V. M., Stothard, S. E., Chipchase, B., Kaplan, C. (2006): Psychosocial outcomes at 15 years of children with a preschool history of speech-language impairment. Journal of Child Psychology and Psychiatry, 47(8), 759-765.

15. Chiat, S. (2001). Mapping theories of developmental language impairment: Premises, predictions and evidence. Language and cognitive processes, 16(2-3), 113-142. 
16. Abu-Rabia, S., and Abu-Rahmoun, N. (2012). "The Role of Phonology and Morphology in the Development of Basic Reading Skills of Dyslexic and Normal Native Arabic Readers." Creative Education 3(7): 1259-1268

17. Abu-Rabia, S., and Taha, H. (2004): Reading and spelling error analysis of native Arabic dyslexic readers. Reading and Writing: An Interdisciplinary Journal, 17(7-8), 651-689.

18. Kieffer, M. J., \& Box, C. D. (2013). Derivational morphological awareness, academic vocabulary, and reading comprehension in linguistically diverse sixth graders. Learning and Individual Differences, 24, 168-175.

19. Bishop, D. and Snowling, M. (2004): Developmental dyslexia and specific language impairment: Same or different? Psychological Bulletin, 1(30), 858- 886.

20. Ullman, M. T., and Pierpoint, E. I. (2005): Specific Language Impairment is not specific to language: The Procedural Deficit Hypothesis. Cortex, 41, 399433.

21. Wise, J. C., Sevcik, R. A., Morris, R. D., Lovett, M. W., \& Wolf, M. (2007): The relationship among receptive and expressive vocabulary, listening comprehension, pre-reading skills, word identification skills, and reading comprehension by children with reading disabilities. Journal of Speech, Language, and Hearing Research, 50, 1093-1109

22. Rack, J.P. (1994): Dyslexia: The Phonological Deficit Hypothesis. In A.J. Fawcett and R.I. Nicolson (Eds.), Dyslexia in Children: Multidisciplinary perspectives. London: HarvesterWheatsheaf.
23. Ginsberg, D., Honda, M., \& O’Neil, W. (2011). Looking beyond English: Linguistic inquiry for English language learners. Language and Linguistics Compass, 5(5), 249-264.

24. Gathercole, S. E., Alloway, T. P., Willis, C., \& Adams, A. M. (2006). Working memory in children with reading disabilities. Journal of experimental child psychology, 93(3), 265-281.

25. Fitzgerald, J., \& Shanahan, T. (2000). Reading and writing relations and their development. Educational Psychologist, 35(1), 39-50.

26. Ouellette, G. P. (2006): What's meaning got to do with it? The role of vocabulary in word reading and reading comprehension. Journal of Educational Psychology, 98(3), 554-566.

27. Taouk, M., \& Coltheart, M. (2004). The cognitive processes involved in learning to read in Arabic. Reading and Writing: An Interdisciplinary Journal, 17, 27-57 .

28. Sukumaran, TU. (2011): Poor Scholastic Performance in Children and Adolescent. Indian Pediatrics. 48(8):597-8

29. Catts, H. W., Adlof, S. M., and Weismer, S. E. (2006): Language deficits in poor comprehends: a case for the simple view of reading. Journal of Speech Language and Hearing Research, 49, 278-293.

30. Alt, M., Hogan, T., Green, S., Gray, S., Cabbage, K., Cowan, N. (2017): Word learning deficits in children with dyslexia. Journal of Speech, Language, and Hearing Research, 60, 1012-1028. 